\title{
ALLOY SPLITTING OF THE Te-DX STATES IN $\mathrm{Al}_{x} \mathrm{Ga}_{1-x} \mathrm{As}$ *
}

\author{
G. Ostermayer, W. Jantsch \\ Institut für Experimentalphysik, Johannes Kepler Universität, 4040 Linz, Austria \\ D. DoBosz, Z.R. ŻYTKIEWICZ AND Z. WILAMOWSKI \\ Institute of Physics, Polish Academy of Sciences \\ Al. Lotnikow 32/46, 00-668 Warszawa, Poland
}

\begin{abstract}
We report investigations of the Hall effect and conductivity of Te doped $\mathrm{Al}_{x} \mathrm{Ga}_{1-x} \mathrm{As}(x=0.3)$. After illumination at low temperature, the conductivity decreases in two steps on warming. These steps are explained in terms of the two sets of energy levels associated with two types of Te-DX centers depending on the neighboring host cation ( $\mathrm{Ga}$ or $\mathrm{Al}$ ) which undergoes the lattice relaxation. The observed persistent increase in mobility is also explained in terms of the two different capture barriers.
\end{abstract}

PACS numbers: 71.55.Eq, 72.80.Ey

The systematic investigation of the static and dynamic electron transport in $\mathrm{Al}_{x} \mathrm{Ga}_{1-x} \mathrm{As}$ shows that the properties of the Te-induced DX centers differ qualitatively from those of the well known Si-induced DX centers [1-4] which was attributed to the qualitatively different configuration coordinate diagrams [5]. In alloys, differences of the local composition lead to a splitting of characteristic donor energies. In this paper we show that much stronger alloy splitting of Te-DX center, as compared to those of Si-DX, leads to some new effects underlying another differences between donors of group IV and of group VI in the III-V compounds. The splitting of the ground DX state together with the temperature dependent position of the conduction band lead to a non-monotonic (step-like) behavior of the temperature dependent carrier concentration close to thermal equilibrium (see Fig. 1). Alloy splitting of the top of the barrier leads to multistage capture process [6]. This very complicated process results in very peculiar photoconductivity. In addition to very well known persistent photoconductivity (PPC), which originates from an electron transfer from DX to conduction band states, a new PPC effect, which originates from a different distribution of electrons among DX states, is observed by a change of the electron mobility.

In the first approximation, the energy of the donor state depends on whether $\mathrm{Ga}$ or $\mathrm{Al}$ nearest neighbor moves to nearest interstitial position in the capture

${ }^{*}$ Work supported by Fonds zur Förderung der Wissenschaftlichen Forschung, Austria, and in Poland by the Committee for Scientific Research under grant No. 204839101. 

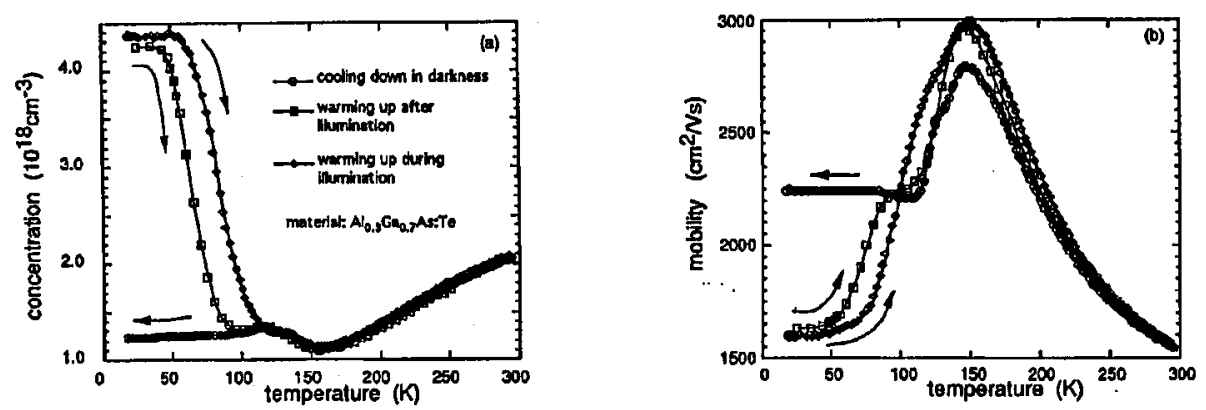

Fig. 1. (a) Temperature dependence of the Hall concentration during cooling and warming with and without illumination and (b) corresponding mobility dependence of an LPE grown $\mathrm{Al}_{x} \mathrm{Ga}_{1-x}$ As sample $\left(N_{\mathrm{Te}}=5 \times 10^{18} \mathrm{~cm}^{-3}, x=0.3\right)$ on top of a thick $\mathrm{Al}_{x} \mathrm{Ga}_{1-x} \mathrm{As}$ buffer and semi-insulating GaAs substrate. For illumination, a light emitting diode $(h \nu=1.36 \mathrm{eV})$ is used.

TABLE

Types of DX centers for group VI donors in $\mathrm{Al}_{x} \mathrm{Ga}_{1-x} \mathrm{As}$.

\begin{tabular}{c|c|c|c|c}
\hline \hline $\mathrm{N}$ & Neighbors & $g_{\mathrm{Al}}(N)$ & $g_{\mathrm{Ga}}(N)$ & Abundance, $p_{N}(x)$ \\
\hline 1 & GaGaGaGa & 4 & 0 & $(1-x)^{4}$ \\
2 & GaGaGaAl & 3 & 1 & $4 x(1-x)^{3}$ \\
3 & GaGaAlAl & 2 & 2 & $6 x^{2}(1-x)^{2}$ \\
4 & GaAlAlAl & 1 & 3 & $4 x^{3}(1-x)$ \\
5 & AlAlAlAl & 0 & 4 & $x^{4}$
\end{tabular}

process which leads to the creation of a DX state [7]. Thus, the simplest approach for the description of the types of the Te-induced DX center in an alloy requires the consideration of at least 5 types of donor centers differing by the number of $\mathrm{Ga}$ and $\mathrm{Al}$ nearest neighbors. Each donor has fourfold degeneracy, $g=g_{\mathrm{Ga}}+g_{\mathrm{Al}}=4$, corresponding to the relaxation of one of the four nearest neighbors in a $\langle 111\rangle$ direction. These states are characterized by two different energies, depending on the type of group III host atom which goes interstitial. From the thermodynamic point of view the various DX states of the same donor must be treated as excited states. The 5 types of donor centers, their degeneracies and relative abundances are specified in Table. This classification does not consider an influence of the next nearest neighbors which would lead to more detailed alloy splitting [3-5].

Our experimental data imply that the height of the barrier for the Te-Ga-DX state is smaller than the height for Te-Al-DX state. As a consequence, electron recapture is more effective for the Te-Ga-DX states. But since their energy of the ground state is higher, as compared with Te-Al-DX state, these states are excited ones. In a temperature range, where the lower barrier is transparent but the higher 
one is not, the excited states of the donor centers can be persistently populated leading to an extra complexity of PPC.

Depending on the temperature range, various types of PPC effects are observed. At very low temperature, when all barriers are non-transparent, all photoexcited electrons gather persistently in the conduction band. In the intermediate temperature range of $50 \mathrm{~K}<T<115 \mathrm{~K}$, the lower barrier (Ga-type) is partially transparent and the conduction electron concentration results from the balance of photoemission and thermal capture [8,9]. The temperature dependence of the quasi-Fermi energy indicates that the energy of the top of this lower barrier is located $120 \mathrm{meV}$ above the bottom of the conduction band. (For Al composition $x=0.3$.) There is, however, no recapture of electrons on the lower Te-Al-DX states which are characterized by the higher barrier.

At higher temperature, $115 \mathrm{~K}<T<140 \mathrm{~K}$, however, the lower (Ga) barrier is practically transparent and the higher (Al) barrier becomes partially transparent leading to a low capture of electrons on the ground Te-Al-DX states. In kinetics, a multistage capture process takes place [6]. In the first stage, a fast capture of conduction electrons on the Te-Ga-DX states occurs. This process is balanced by the effective re-emission. Next, the much slower process of electron recapture across the higher barrier of the lower Te-Al-DX states takes place.

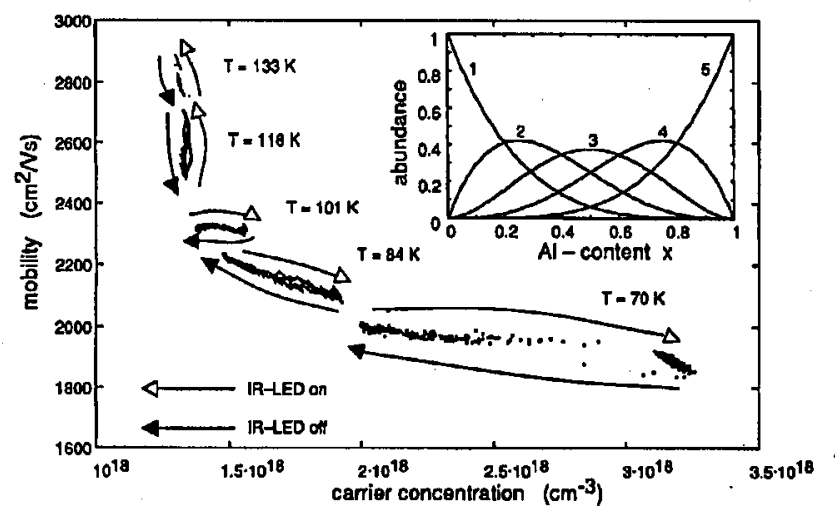

Fig. 2. Electron mobility plotted vs. the Hall concentration as obtained from various isothermal transients after switching the light on and off. Two types of variation are distiguishable. For $T<110 \mathrm{~K}$, the mobility is almost independent of the carrier concentration $n$, which shows normal PC. For higher $T$, in contrast, only the mobility is affected by illumination. The insert shows the relative abundancies of different DX centers as a function of Al composition $x$.

Peculiarities of the photoconductivity kinetics (PC) are seen in Fig. 2 where the mobility is plotted vs. electron concentration for various isothermal PC transients. Two types of the PPC can be easily distinguished: (i) in the free carrier concentration and (ii) in their mobility. In the higher temperature range, there is almost no variation of the electron concentration. Most of the conductivity varia- 
tion there comes from the variation of the electron mobility. The lack of changes in the electron concentration can be explained in terms of transfer of the electron from one to another state of the impurity center. Such transfer does not influence the concentration of the mobile electrons. The details of the statistics of the donor center are shown in Table. For an alloy with a composition bigger than $15 \%$ of $\mathrm{Al}$, the capacity of the Te-Al-DX states is big enough to bind all electrons in the system (each DX donor state binds 2 electrons) and one can assume that close to thermal equilibrium only the ground (Te-Al-DX) states are occupied. Most of these states have at least one excited Ga-DX state. Thus the photoexcitation effectively transfers electrons mainly to the excited states of the same center. The phototransfer of electrons to the same donor, which does not have an Te-Al-DX ground state, does not affect the carrier concentration but it influences the impurity self-screening and thus the electron mobility $[6,10]$.

Because of the very complicated multistage process, the height of the $\mathrm{Al}$ barrier is difficult to be determined directly from the experiment. A comparison of the temperature ranges of various types of the PPC allows, however, to estimate that the top of the (one-electron) barrier is located at about $15-20 \mathrm{meV}$ above the top of the Ga-DX barrier.

\section{References}

[1] W. Shan, P.Y. Yu, M.F. Li, W.L. Hansen, E. Bauser, Phys. Rev. B 40, 7831 (1989).

[2] J.E. Dmochowski, L. Dobaczewski, W. Jantsch, J. M. Langer, Phys. Rev. B 40, 9671 (1989).

[3] L. Dobaczewski, P. Kaczor, M. Missous, A.R. Peaker, Z. Żytkiewicz, Phys. Rev. Lett. 68, 2508 (1992).

[4] R. Piotrzkowski, E. Litwin-Staszewska, T. Suski, L. Konczewicz, J.L. Robert, W. Stankiewicz, J. Appl. Phys. 73, 2572 (1993).

[5] Z. Wilamowski, T. Suski, W. Jantsch, Acta Phys. Pol. A 82, 561 (1992).

[6] Z. Wilamowski, W. Jantsch, G. Ostermayer, J. Kossut, Mater. Sci. Forum 83-87, 805 (1992).

[7] D.J. Chadi, K.J. Chang, Phys. Rev. Lett. 61, 873 (1988) and Phys. Rev. B 39, 10063 (1989).

[8] W. Jantsch, Z. Wilamowski, G. Ostermayer, Mater. Sci. Forum 83-87, 799 (1992).

[9] Z. Wilamowski, W. Jantsch, G. Ostermayer, Acta Phys. Pol. A 80, 283 (1991).

[10] Z. Wilamowski, J. Kossut, T. Suski, P. Wisniewski, L. Dmowski, Semicond. Sci. Technol. 6, B34 (1991). 